\title{
Modified Wilkinson Power Dividers for Millimeter-Wave Integrated Circuits
}

\author{
Stephen Horst, Student Member, IEEE, Ramanan Bairavasubramanian, Student Member, IEEE, \\ Manos M. Tentzeris, Senior Member, IEEE, and John Papapolymerou, Senior Member, IEEE
}

\begin{abstract}
A modification of the Wilkinson power divider is presented that eases planar implementation while maintaining performance. By adding transmission lines between the resistor and the quarter-wave transformers of the traditional design, a range of valid solutions exists that meet the conditions of being reciprocal, isolated between the output ports, and matched at all ports. The proposed design is particularly useful at millimeter-wave frequencies where reduced physical dimensions make a circuit configuration suitable for low-cost package-level implementation difficult using traditional methods. Two frequency bands are demonstrated. At $V$-band, the circuit gives 0.3 -dB excess insertion loss, 19-dB isolation, and $50 \%$ bandwidth. At the $W$-band, the circuit gives 0.75-dB excess insertion loss, 24-dB isolation, and $39 \%$ bandwidth.
\end{abstract}

Index Terms-Balun, hybrid, millimeter-wave circuits, power divider, Wilkinson power divider.

\section{INTRODUCTION}

$\mathbf{T}$ HE NEED to divide an input signal into several identical output copies is common among many communication systems. Often there is a necessity to isolate the output ports of such a divider so that mismatches and reflected signals on one output port do not propagate to other paths in the system. An elegant solution to this problem is the Wilkinson power divider [1], [2]. Developed in the early 1960s, this hybrid power divider utilizes resistors between output ports in order to provide isolation over a given frequency band. Despite its clever use of symmetry, the traditional Wilkinson divider suffers from several drawbacks when implemented in a planar form such as the printed wiring board techniques ubiquitous to present day circuit technology. The original Wilkinson power divider was designed for use in shielded coaxial systems at frequencies low enough not to warrant consideration of additional parasitic effects caused by geometric inconsistencies in the physical layout of the circuit. In RF and millimeter-wave systems, these effects can be devastating to the performance of the circuit. This paper presents a modified Wilkinson power divider circuit that achieves similar performance, while better suited to the layout considerations of a planar environment.

Manuscript received May 27, 2007; revised August 14, 2007.

S. Horst, M. M. Tentzeris, and J. Papapolymerou are with the School of Electrical and Computer Engineering, Georgia Institute of Technology, Atlanta, GA 30332 USA (e-mail: shorst@gatech.edu).

R. Bairavasubramanian was with the School of Electrical and Computer Engineering, Georgia Institute of Technology, Atlanta, GA 30332 USA. He is now with Skyworks Solutions Inc., Cedar Rapids, IA 52411 USA.

Color versions of one or more of the figures in this paper are available online at http://ieeexplore.ieee.org.

Digital Object Identifier 10.1109/TMTT.2007.908672

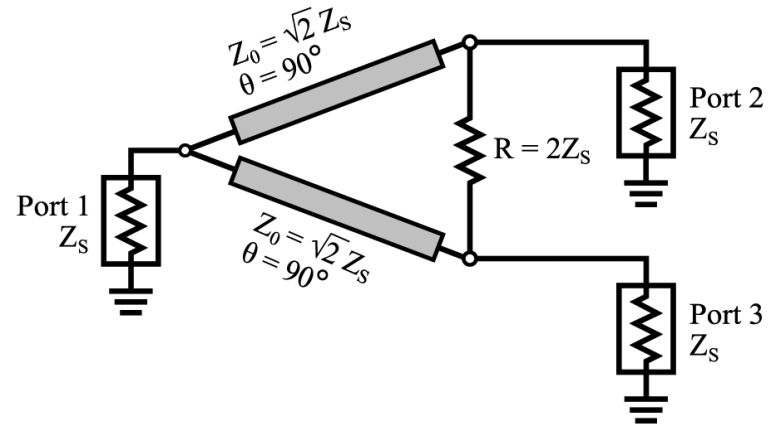

Fig. 1. Traditional Wilkinson design. From [1].

Shown schematically in Fig. 1, the traditional Wilkinson power divider can suffer from parasitic effects at high frequencies that can significantly inhibit performance in an integrated planar design. These parasitics typically derive from undesired coupling between the two quarter-wave arms, or distributed effects introduced by the physical requirements of stretching the resistor between the two arms. Integrated resistors introduce far fewer parasitic effects than resistors contained in a separate package, but its dimensions still cause it to take on the characteristics of a transmission line at high frequencies. Typical solutions to these problems have involved wrapping the quarter-wave arms into rounded shapes that converge briefly for resistor placement using the smallest resistor footprint possible [3]-[5]. Due to the necessary bends, it is often difficult to incorporate these designs at the package level for millimeter-wave frequencies because of the small length-to-width ratio of the quarter-wave transformers. On the other hand, on-chip implementation of the transformers is large compared with the available active components, leading to more space-effective topologies implemented with active devices replacing the transmission lines [6]. Another solution uses additional half-wave transformers to connect a small resistor between widely separated arms, which is compatible with the design procedure presented here, although this method greatly increases the size of the circuit [7]. This paper demonstrates a method to achieve an optimal solution using a simple and easily realizable structure that requires little optimization and a small physical area. Since there are no complex bends, the design also scales well throughout most RF and millimeter-wave frequencies.

\section{CirCUit ANALYSIS}

The design presented in this research, shown in Fig. 2, extends the original Wilkinson design by adding additional 


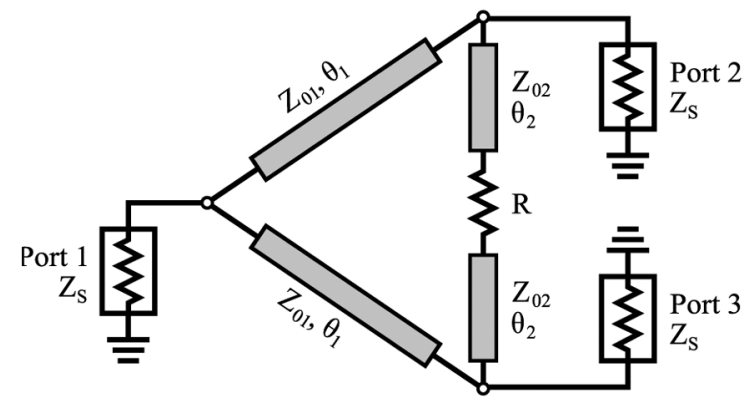

Fig. 2. Proposed modification of the Wilkinson design.

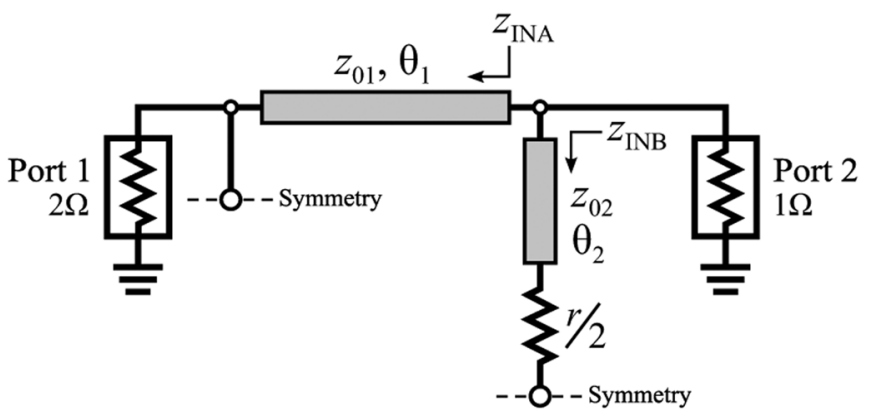

Fig. 3. Symmetric reduction of the circuit. All impedances have been normalized to the system impedance.

transmission line elements between the quarter-wave transformer arms of the divider. This addition creates the framework for an object with geometric dimensions in the space between the quarter-wave transformers and the ideal resistor, which can, in turn, be used to increase the spacing between the arms; reducing the coupling between them. These added transmission lines can be implemented either as standalone elements, or absorbed into the integrated thin film resistor, or a combination of the two. By absorbing the physical area of the resistor into the transmission lines, the circuit can decrease the sensitivity to distributed effects seen in integrated resistors since the resistor becomes a natural extension of the added line. As will be demonstrated, the proposed design maintains the same matched, reciprocal, and isolated response seen in the Wilkinson power divider.

Like the traditional Wilkinson divider, the key to this design lies in its symmetry. By dividing the circuit in half along its line of symmetry and using odd- and even-mode analysis, the complexity of the analysis can be greatly reduced. The reduced circuit is illustrated in Fig. 3. The line of symmetry bisects the resistor $R$, which can be represented as two resistors in series with a value of $R / 2$. Likewise, the impedance of the input port can be divided in parallel, doubling the effective impedance of the port. All impedances, including the resistor $R$, have been normalized to the system impedance throughout this derivation, and are hereafter denoted by lowercase letters to reflect this fact. The circuit then contains five unknowns, which are the characteristic impedance and electrical length of the "arm" segments, denoted as $z_{01}$ and $\theta_{1}$, respectively, the characteristic impedance and electrical length of the "stub" segments, denoted as $z_{02}$ and $\theta_{2}$, respectively, and the value of the resistor $r$.

\section{A. Even-Mode Analysis}

In the even mode, the symmetric ports are excited with currents of equal magnitude and phase. Since the currents are in the same direction, a virtual open is created along the line of symmetry, which eliminates the resistor from the circuit. The circuit then becomes a simple two-port matching network. Looking into port 2, we can solve for the impedance of each path, denoted as $z_{I N A}$ and $z_{I N B}$ in Fig. 3

$$
\begin{aligned}
& z_{I N A}=z_{01} \frac{2+j z_{01} \tan \left(\theta_{1}\right)}{z_{01}+j 2 \tan \left(\theta_{1}\right)} \\
& z_{I N B}=-j z_{02} \cot \left(\theta_{2}\right) .
\end{aligned}
$$

The parallel combination of these impedances should equal the port impedance in order to be matched. Adding the admittances $y_{I N A}$ and $y_{I N B}$ gives

$$
\frac{z_{01}+j 2 \tan \left(\theta_{1}\right)}{2 z_{01}+j z_{01}^{2} \tan \left(\theta_{1}\right)}+j \frac{\tan \left(\theta_{2}\right)}{z_{02}}=1
$$

which can be expanded and separated into two complex equalities. The real part of the equation yields

$$
z_{01} z_{02}=2 z_{01} z_{02}+z_{01}^{2} \tan \left(\theta_{1}\right) \tan \left(\theta_{2}\right)
$$

which simplifies to

$$
-\frac{z_{02}}{z_{01}}=\tan \left(\theta_{1}\right) \tan \left(\theta_{2}\right)
$$

The imaginary part of the equation becomes

$$
2 z_{02} \tan \left(\theta_{1}\right)=z_{01}^{2} z_{02} \tan \left(\theta_{1}\right)-2 z_{01} \tan \left(\theta_{2}\right)
$$

which can also simplify, giving

$$
1-\frac{z_{01}^{2}}{2}=\frac{1}{\tan ^{2}\left(\theta_{1}\right)} \text {. }
$$

Since this is a passive matching network, the circuit will be reciprocal, and analysis from port 1 will yield the same result.

\section{B. Odd-Mode Analysis}

In the odd mode, the symmetric ports are once again excited with currents of equal magnitude, but this time with opposite phases, creating a virtual short along the line of symmetry. Since the circuit is symmetric through port 1, the short will appear across the port, eliminating it from the circuit. The short will also appear at the base of the bisected resistor $R / 2$, leaving it to absorb all the incident power in order to maintain the matched condition. Using the same method described for the even-mode analysis, we can obtain the matching condition

$$
\frac{z_{02}+j \frac{r}{2} \tan \left(\theta_{2}\right)}{z_{02} \frac{r}{2}+j z_{02}^{2} \tan \left(\theta_{2}\right)}-j \frac{\cot \left(\theta_{1}\right)}{z_{01}}=1
$$


This equation can also be expanded and given as a set of complex equalities, leaving a real equality that simplifies to

$$
1-\frac{r}{2}=\tan ^{2}\left(\theta_{2}\right)
$$

and an imaginary equality that simplifies to

$$
r=z_{02}^{2}
$$

If we apply (10) to (9), it can easily be shown that

$$
1-\frac{z_{02}^{2}}{2}=\tan ^{2}\left(\theta_{2}\right)
$$

which is a complementary equation to (7). Therefore, if we were to substitute (7) and (11) into (5), an equation relating $z_{01}$ and $z_{02}$ can be obtained and simplified to show that

$$
z_{01}=z_{02}
$$

Using this knowledge, (5) simplifies to

$$
\tan \left(\theta_{1}\right) \tan \left(\theta_{2}\right)=-1
$$

which implies that $\theta_{1}$ and $\theta_{2}$ must be orthogonal. We are now left with four normalized equations to describe a circuit with five unknowns

$$
\begin{aligned}
z_{01} & =z_{02} \\
r & =z_{02}^{2} \\
\theta_{2} & =\tan ^{-1}\left(\sqrt{1-\frac{r}{2}}\right) \\
\theta_{1} & =\frac{\pi}{2}+\theta_{2} .
\end{aligned}
$$

This under-determined system of equations will give a range of solutions that meet the requirements of being matched, reciprocal, and isolated between the output ports. The range of several variables in this system are bounded by physical conditions imposed by $r$ and (16). In order to be a physically realizable circuit, $\theta_{2}$ must be real and $r$ must be positive. Enforcing these conditions in the normalized circuit, it can easily be shown that $r \in(0,2]$, which implies $z_{01}, z_{02} \in(0, \sqrt{2}]$. It can also be shown that $\theta_{2} \in[n \pi, n \pi+(\pi / 4))$, where $n$ is any integer. If we allow $\theta_{2}$ to go to 0 , we see that the system reduces to the familiar Wilkinson design.

\section{Circuit Design}

Although the preceding theory is applicable to any frequency, the practical advantages for this design are best seen at millimeter-wave frequencies. At lower RF frequencies below around $10 \mathrm{GHz}$, fabrication tolerances and transmission line length-to-width ratios are generally forgiving enough to fold the arms of the standard Wilkinson divider, where $\theta_{2}=0$, to provide a smaller size and greater bandwidth than other variants of the proposed design. However, as the wavelength decreases, the electrical length of the transmission lines shrink, while the

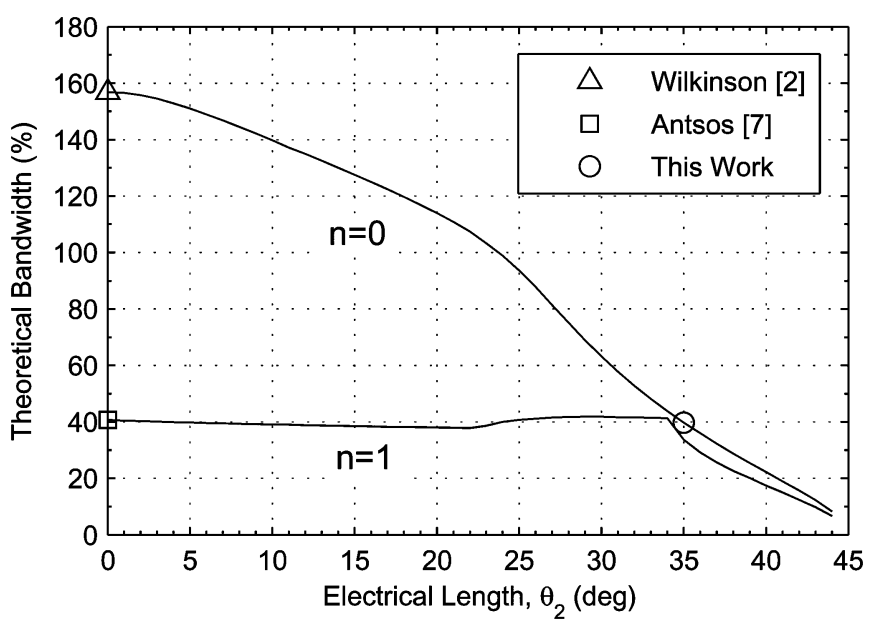

Fig. 4. Theoretical bandwidth based on ideal simulations using a $2: 1$ VSWR rubric. The trace represents the range of solutions given by the proposed design procedure across two periods, while the individual markers indicate compatible published variants from [2] and [7], and the variant demonstrated in this study. The length of $\theta_{2}$ is represented across its valid range $[n \pi, n \pi+(\pi / 4))$ for $n=0$ and $n=1$.

width determining the characteristic impedance remains close to constant. This combination of changing dimensions makes the physical layout of these bending techniques impractical at millimeter-wave frequencies. It is here where the simple structure and slightly larger size of the proposed design are advantageous. Antsos [7] presents a similar structure that, like the traditional Wilkinson power divider, is a specific case of the broader theory presented here. However, as this circuit is merely a multiple of the traditional Wilkinson divider $\left(\theta_{2}=\pi\right.$ in this case), the circuit is unnecessarily large. As shown in the equations derived in Section II, adding a small length of transmission line between the resistor and the quarter-wave transformer reduces the necessary characteristic impedance of the quarter-wave transformers themselves. Their length increases at the same time, leading to a range of solutions near the middle of the valid range that maximize the length-to-width ratio of these elements. As with all transmission lines, the initial dimensions and the rates at which these changes occur are highly dependent on the substrate used. The various factors that must be considered during the design process to achieve the best solution for a given situation are explored here.

Selecting the proper variant of this design methodology involves a tradeoff analysis between performance, size, and a variety of design rules dependent on the technology used to implement the design. One aspect to consider when adding additional lengths of transmission line to a circuit is the bandwidth. The proposed design procedure is capable of producing a variety of wide circuit bandwidths, as shown in Fig. 4. Maximum bandwidth is achieved by keeping line lengths to a minimum, as in the original Wilkinson design. As additional length is added to the lines based on the theory presented here, bandwidth decreases according to the $n=0$ trace shown in Fig. 4 . Due to the periodic nature of electrical lengths in transmission lines, multiples of the valid range will also produce valid solutions. The first multiple, denoted by $n=1$, is plotted in Fig. 4 . Note that the bandwidth response in this region remains relatively flat up to an unwrapped electrical length of approximately $35^{\circ}$ before 
falling off. However, the bandwidth of almost any of these configurations exceeds that of many other common system components, even when reduced to levels below this plateau. Therefore, the design variant fabricated and measured in this study was chosen to emphasize the ability of the added stubs to enable compact and robust millimeter-wave power dividers using low-cost technology rather than achieving the widest bandwidth possible. The configuration chosen for this purpose is denoted by the circle marker in Fig. 4 for reasons that will be clarified shortly.

Recall that the primary motivation behind this design involves the reduction of both coupling between the parallel arms and unwanted distributed effects appearing across the integrated resistor. The effects of coupling between two microstrip lines are well understood [8]. However, the specific amount of coupling is highly dependent on the properties and dimensions of the substrate being used, which makes it difficult to quantify how much will be present in a general scenario. Generally speaking, proximity is the primary cause of undesired coupling. Therefore, if the coupling between the impedance transformers is determined to be too strong, adding additional length to the stub $\left(\theta_{2}\right)$ effectively separates the paths of concern and reduces the problem. As previously mentioned, this is most useful at high frequencies where introducing bends into the lines is impractical.

A problem that typically has a greater impact on the performance of the circuit involves the parasitics of the resistor. Resistors, whether they are integrated or packaged, occupy physical space that is not accounted for in the traditional Wilkinson model. When an RF designer implements a design that must contend with one of these components, such as a traditional Wilkinson power divider, the result is typically that hand calculations produce unusable baseline results and an electromagnetic (EM) field solver must be used to obtain a useful solution. By using the proposed design, the parasitics of the resistor can be absorbed into the extra stub length between the resistor and impedance transformers, paving the way for accurate results using simple hand calculations.

When integrated onto the substrate, a resistor is little more than a transmission line with a given loss per unit length. In addition, many integrated resistor materials have a very low sheet resistance value, meaning the resistor must be relatively long in order to achieve the desired value. This only increases these associated transmission line parasitics. Since even at millimeter-wave frequencies the resistor length is still small with respect to the wavelength, the resistor can be modeled as a series combination of transmission lines and a resistor, as shown in Fig. 5. These parasitic lines can then be easily absorbed into the stub extensions of the proposed design.

The layout of the proposed divider can be seen in Fig. 6 . By keeping the structure simple without unnecessary bends, the layout can be scaled very small to accommodate high-frequency designs where the required transmission line lengths are not much larger than the necessary widths. The simple design also means far fewer parasitic modeling effects need to be considered. As previously described, the resistor dimensions can be absorbed into $\theta_{2}$ using the model in Fig. 5. This leaves only three $\mathrm{T}$-junctions present in the layout that are as yet unaccounted for in the design. These junctions are each denoted by an " $\mathrm{X}$ " in

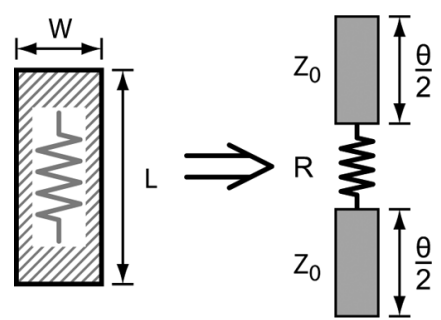

Fig. 5. Equivalent high-frequency model of an integrated resistor used in the proposed design.

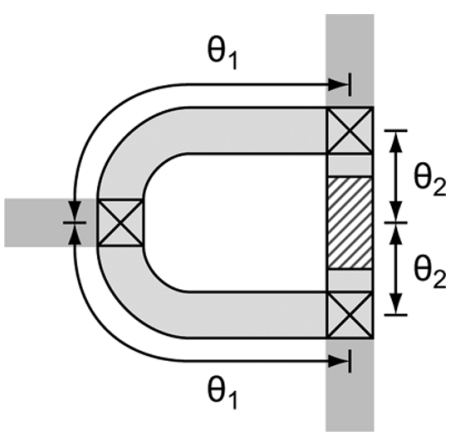

Fig. 6. General layout of the proposed power divider. The resistor dimensions and $\mathrm{T}$-junctions can be absorbed into the transmission line lengths for accurate results using hand calculations.

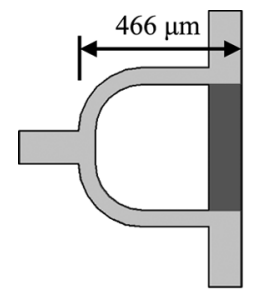

(A)

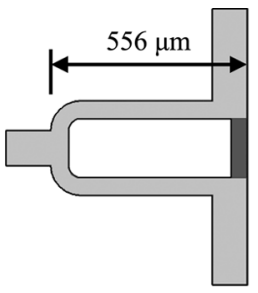

(B)

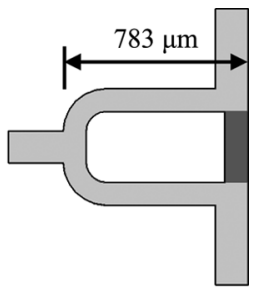

(C)
Fig. 7. Variations on power divider design at $W$-band. (A) Traditional design using a large resistor. (B) Traditional design using a small resistor. Note that although the overall size of the resistor has changed, the length-to-width ratio and, therefore, the resistance used in (A) and (B) are the same. (C) Designed using the equations presented here, using the solution where the resistor dimensions coincide with the necessary stub dimensions.

Fig. 6. A rough approximation that works well for hand calculations involves absorbing half the length of each T-junction into the length of each line attached to the "T," as shown by the lengths of $\theta_{1}$ and $\theta_{2}$ in Fig. 6.

A comparison between traditional Wilkinson designs and the design procedure proposed here can be used to illustrate the effectiveness of this technique. Fig. 7 shows the layout of several power dividers at the $W$-band in a standard $50-\Omega$ system. Fig. 7(A) is a traditional Wilkinson design measuring $466 \times 568$ $\mu \mathrm{m}^{2}$ that uses a large resistor to reduce coupling between the quarter-wave transformers, but in turn incurs greater resistor parasitics. Fig. 7(B) is a similar design using a small resistor to minimize the parasitics of the resistor, but increases the coupling between the quarter-wave transformers. This circuit occupies an area of $556 \times 253 \mu \mathrm{m}^{2}$. Also shown in Fig. 7(C) is a layout from a comparable circuit designed using the proposed method that accounts for the physical dimensions of the resistor. The solution used was selected by simultaneously solving for the value 

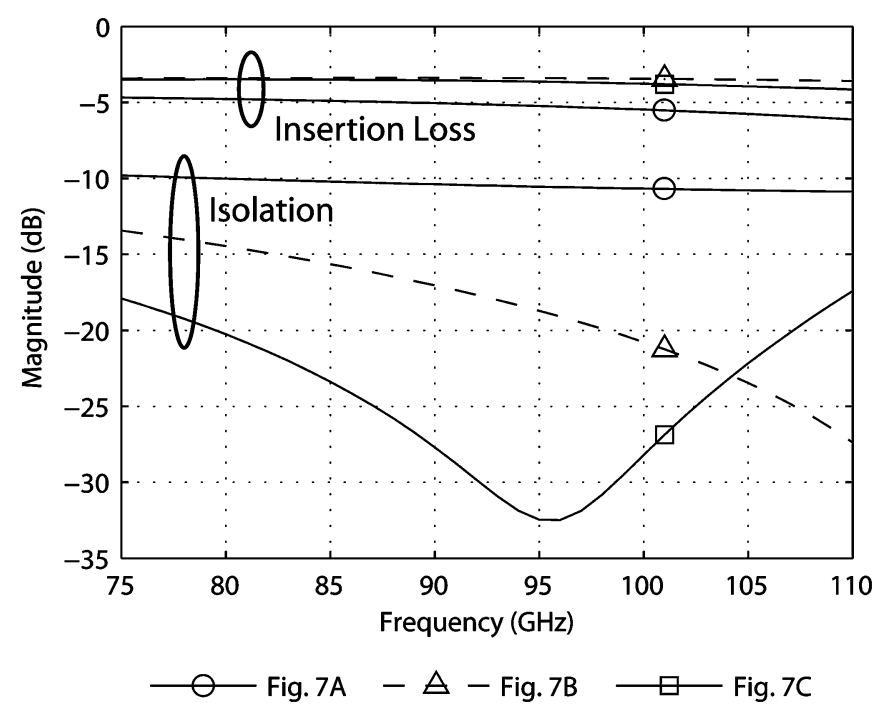

Fig. 8. Simulation results comparing the traditional Wilkinson design with the proposed technique.

where the required resistor length-to-width ratio matched the characteristic impedance and phase needs of the transmission lines. This solution is highly dependent on the sheet resistance and substrate characteristics used in the circuit. For a sheet resistance of $25 \Omega$ /square, and a substrate with a thickness of $50 \mu \mathrm{m}$ and dielectric constant of 3 , this solution occurred at a stub length of $\theta_{2}=23.5^{\circ}$. Computing the rest of the design variables resulted in a circuit measuring $783 \times 397 \mu \mathrm{m}^{2}$, approximately $17 \%$ larger than the traditional design shown in Fig. 7(A). It is interesting to note that if an optimization routine were to be run minimizing the return loss and isolation on the traditional designs with the resistor, linewidth, and line length allowed to vary, the values of each parameter would approach the solution shown in Fig. 7(C).

Simulation results of the insertion loss and isolation performance using Ansoft's High Frequency Structure Simulator (HFSS) for each layout are shown in Fig. 8. These results demonstrate that resistor parasitics have a much larger effect on circuit performance compared to parasitic coupling in this situation. This is expected for a thin substrate with a low dielectric constant such as the one used here. Although the design in Fig. 7(B) provides adequate results, the small resistor size will increase the variation seen during fabrication. The design using the proposed technique offers superior isolation across most of the $W$-band and similar insertion-loss performance at sizes that are more appropriate for fabrication. The bandwidth of the circuit in Fig. 7(C) is $85.9 \%$, down slightly from the theoretical value predicted in Fig. 4. Likewise, the $134.8 \%$ bandwidth of the traditional circuit using the small resistor was down from its predicted value of nearly $160 \%$. These reduced numbers are attributed to substrate and conductor losses that were not accounted for in the theoretical prediction. The traditional design using the large resistor never achieved a voltage standing-wave ratio (VSWR) greater than $2: 1$ on the input port and, therefore, no bandwidth estimate could be made. Considering these results, the proposed design is most useful for millimeter-wave applications on low-cost package-level technologies. In such situations, the circuit provides superior isolation and similar insertion-loss characteristics compared to a traditional Wilkinson power divider. More importantly, it can be used to relax feature size requirements of the resistor and quarter-wave transformer elements to the extent that the power divider will conform to the design rules of most package level fabrication processes; a feature that will be explored in Section IV.

The equations presented here provide a range of solutions that can be tailored to fit any number of applications. One of these solutions selected for further study occurs when the normalized value of the resistor is 1 . At this value, the normalized characteristic impedance of all the transmission lines used in the circuit are also 1. This configuration is ideal because there is no transition necessary to interface with the rest of the system, as all of the port lines will already be the same size as the system impedance lines feeding the circuit. Using the middle value in the possible range of $r$ is also beneficial because it eliminates the physical extremes seen at both ends of the spectrum. At the one end is the traditional Wilkinson design, which provides narrow transformer lines and high-resistance values with very little tolerance for transmission line stub lengths. The drawbacks of this design at high frequency have been discussed at length. At the other end of the spectrum, the transformer lines and resistor have values so low that the physical restrictions reverse, with transmission linewidths becoming extremely wide compared to its length, resulting in unrealistic geometries. The degree of these effects are dependent on the thickness and dielectric constant of the substrate. Maintaining a high length-to-width ratio in the added stubs will also allow for the use of lower sheet resistance materials since longer resistors can be used. Using these criteria to relax the physical dimensions of both the impedance transformer and resistor, while maintaining a reasonable geometry and minimizing parasitics to demonstrate the robust nature of the design, has led to the selection of the variant where normalized $z_{0}=1$ to confirm the performance of this design procedure.

\section{MEASuREMENT AND Results}

Two different millimeter-wave frequency bands were tested to demonstrate this design using the variant of interest where $r, z_{01}$, and $z_{02}$ all have normalized values of 1 . These values necessitate the electrical lengths $\theta_{1}$ and $\theta_{2}$ to be $125.26^{\circ}$ and $35.26^{\circ}$, respectively, in order to satisfy (14)-(17). The first iteration was designed at $60 \mathrm{GHz}$ in the $V$-band and the second at $90 \mathrm{GHz}$ near the center of the $W$-band. The designs were fabricated on a liquid crystal polymer (LCP) substrate with a thickness of $50 \mu \mathrm{m}$. LCP is a low-cost organic substrate that has been shown to give excellent performance at millimeter-wave frequencies [9].

The integrated resistors were achieved through the use of foils, where a $0.5-\mathrm{oz}$ sheet of copper is sold with a pre-deposited NiCr thin film on one side. This foil can then be laminated to the substrate and formed into individual traces and resistors through standard photolithography and chemical etching [10]. The advantage of this process is that thin films can be used for low-cost large-area processing without having to resort to expensive vacuum deposition techniques. A major draw- 


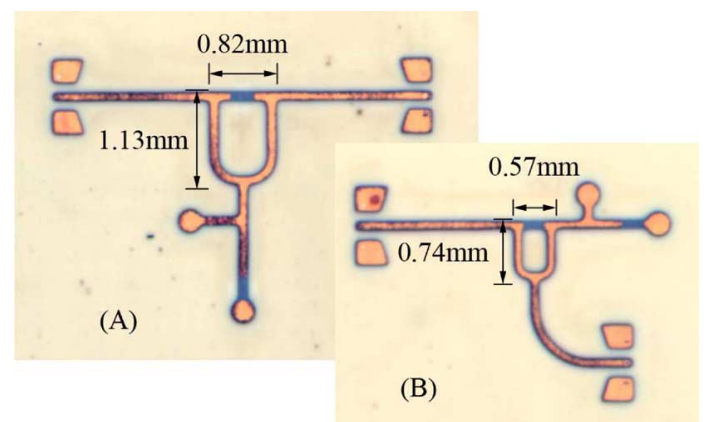

Fig. 9. Fabricated power divider test structures. (A) $V$-band isolation structure with a termination connected to the input port. (B) $W$-band thru structure with a termination on one of the output ports.

back to using this method, however, is that with the thick $18-\mu \mathrm{m}$ copper layer, needed to provide stability to the foil before lamination, the minimum reliable feature size is around $75 \mu \mathrm{m}$. This restriction demonstrates the advantage of using this variant of the design as opposed to the traditional Wilkinson design. A 50 - $\mu$ m-thick substrate with a dielectric constant of 3 requires a $113-\mu \mathrm{m}$-wide line for a $50-\Omega$ characteristic impedance, while a 70.7- $\Omega$ line needs to be $57-\mu \mathrm{m}$ wide. While the low-cost technology implemented here is not capable of producing the narrow $70.7-\Omega$ lines required by the traditional Wilkinson design, it can produce the $50-\Omega$ lines needed by this variant.

Foils with a $25-\Omega$ /square sheet resistance were used for the integrated resistors. This relatively low value necessitates a resistor with a length of at least two squares in order to implement most useful variants of the design, which is more than adequate to make the distributed effects of the resistor apparent. The fabricated power dividers can be seen in Fig. 9. The $V$-band design measures $1.13 \times 0.82 \mathrm{~mm}$, while the $W$-band design measures $0.74 \times 0.57 \mathrm{~mm}$. Due to the additional line lengths involved, the circuit is slightly larger than the traditional Wilkinson power divider, but saves significant space compared to the design presented by Antsos in [7], which would be used for similar applications. Comparing the $W$-band design presented here to a similar design based on the Antsos variant, an area reduction of over $80 \%$ is achieved.

Since the power divider is a three-port device, and a two-port network analyzer needed to be used to take measurements, a solution for terminating the third unmeasured port needed to be found. The easiest method proved to be creating custom $50-\Omega$ terminations using a field solver simulation to determine the impedance of an integrated resistor at the design frequency. These resistors were readily available since they were already required for the power divider. A simple matching network was then used to bring the impedance to $50 \Omega$, the system impedance of the measurement system. This process produced terminations accurate enough to cover the design bands of the circuits. These structures can be seen attached to the fabricated dividers in Fig. 9. Measurements indicate the $V$-band terminations have better than 2:1 VSWR across the entire $50-75-\mathrm{GHz}$ band, while the $W$-band terminations meet this specification from 78 to $103 \mathrm{GHz}$.

Two different design iterations were performed at each frequency band. The first used only hand calculations to derive the

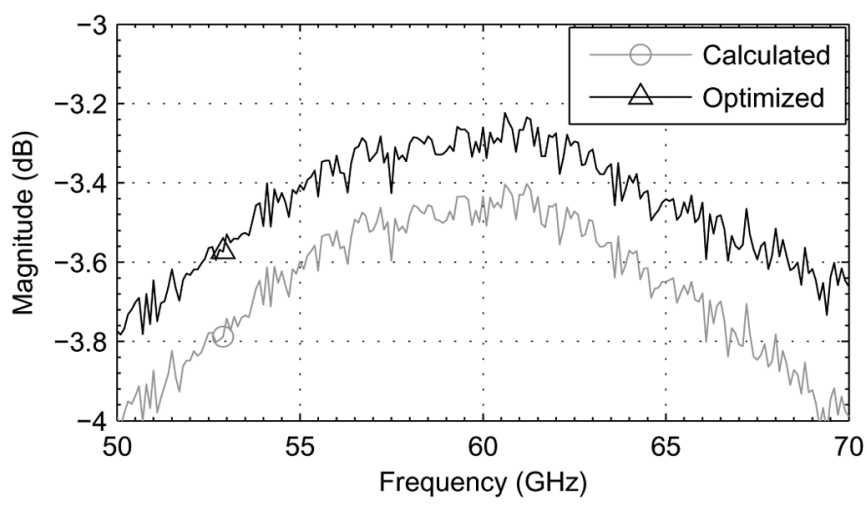

Fig. 10. $\quad V$-band insertion-loss measurements. Optimization improves the loss by $0.2 \mathrm{~dB}$ over basic hand calculations.

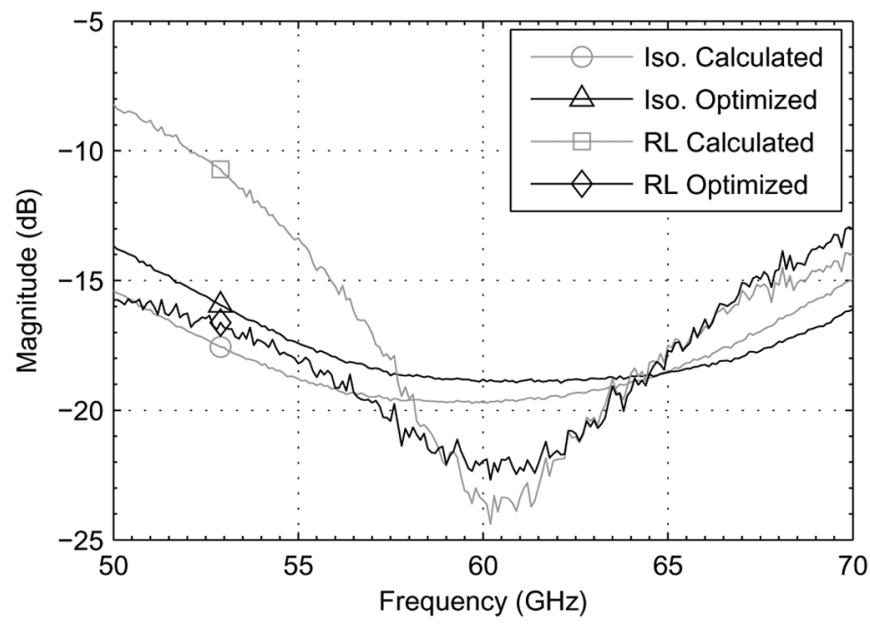

Fig. 11. $V$-band isolation and input return-loss measurements. Optimization provides little improvement over hand calculations.

dimensions of the divider. The second then modeled and optimized the design in a commercial EM field solver. An optimization scheme setting minimum targets for $S_{11}, S_{22}$, and $S_{32}$ at the design frequency was selected. The measured performance of these designs can be seen in Figs. 10-13. The insertion loss of the $V$-band circuit is $0.3 \mathrm{~dB}$ below the ideal value of $3 \mathrm{~dB}$ at the design frequency when optimized, and the isolation is approximately $15 \mathrm{~dB}$ across the band. Optimization provided a $0.2-\mathrm{dB}$ improvement in the insertion loss, but no improvement in isolation when compared to hand calculations. The $W$-band circuit shows $0.75-\mathrm{dB}$ excess insertion loss at the design frequency, also with an isolation around $15 \mathrm{~dB}$ across the band. Here, optimization gives no improvement over hand calculations with respect to insertion loss, but the isolation is improved by $5 \mathrm{~dB}$ in the center of the band and corrects a $15 \%$ frequency shift seen in the return-loss measurements.

These results show that while optimization with an EM field solver may provide some improvement in the response of the circuit, for the most part, the effect of these complex calculations is minimal compared with simple hand calculations. Again, defining the bandwidth as the region where the circuit shows better than 2:1 VSWR on the input port, measured results at the $W$-band show a $38.9 \%$ bandwidth, very close to the value predicted in Fig. 4 . The bandwidth at the $V$-band 


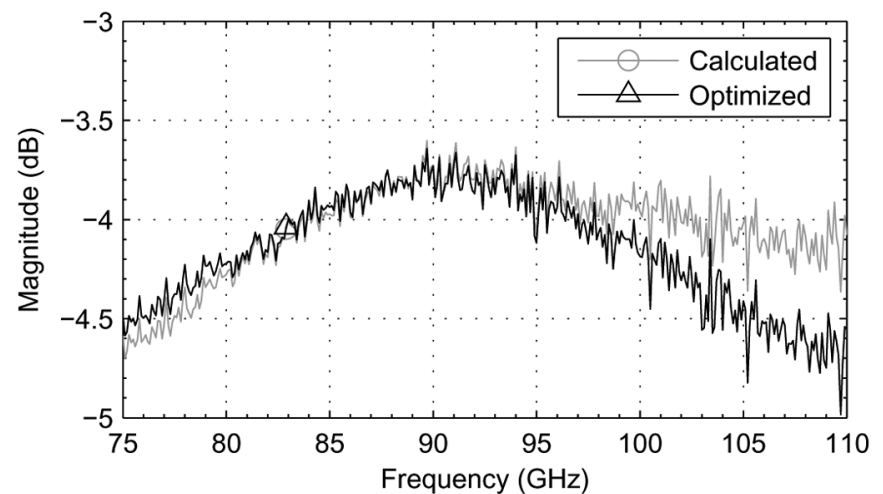

Fig. 12. $W$-band insertion loss measurements. Optimization provides no improvement over hand calculations.

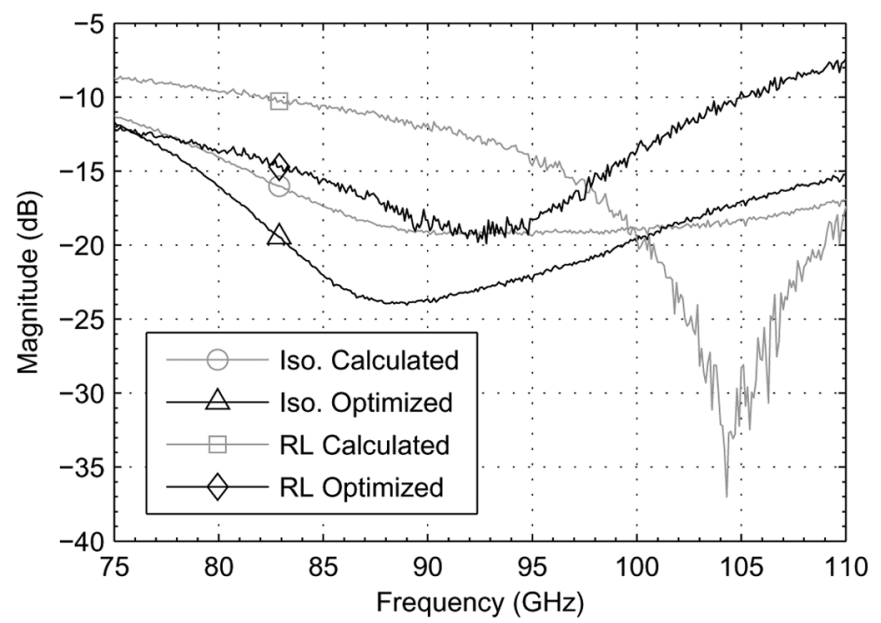

Fig. 13. $W$-band isolation and input return-loss measurements. Optimization improves the isolation by $5 \mathrm{~dB}$ and corrects a frequency shift seen in the return loss.

was around $50 \%$, which well exceeded the predicted value. Even at millimeter-wave frequencies, this design is very robust because it is much better adapted to a planar form factor than the original Wilkinson divider. The results compare well with previously published power dividers at the $W$-band [3], [5] with the additional benefit of being well suited for low-cost technologies where thicker substrates and larger feature sizes make millimeter-wave designs difficult.

\section{CONCLUSION}

An extension of the traditional Wilkinson power divider has been presented. The addition of transmission lines between the resistor and quarter-wave transformers has been shown to absorb the high-frequency parasitics typically unaccounted for in traditional designs, while maintaining the ideal performance characteristics of being matched, reciprocal, and isolated between the output ports. The prototype designs show excellent performance at millimeter-wave frequencies, even when implemented using low-cost technology typically reserved for lower frequency circuits with less critical dimensions. The design has also been demonstrated to be very robust with simple hand calculations providing reliable dimensions for near-optimal circuit performance. Using the design procedure presented, millimeter-wave power dividers are no longer restricted to depend on vacuum deposition techniques, and can become accessible to a wider market.

\section{ACKNOWLEDGMENT}

The authors would like to thank the Rogers Corporation, Rogers, CT, and Ticer Technologies LLC, Chandler, AZ, for supplying the raw materials used in this research.

\section{REFERENCES}

[1] D. M. Pozar, Microwave Engineering, 3rd ed. New York: Wiley, 2005.

[2] E. J. Wilkinson, "An $N$-way hybrid power divider," IEEE Trans. Microw. Theory Tech., vol. MTT-8, no. 1, pp. 116-118, Jan. 1960.

[3] K. J. Herrick and L. P. B. Katehi, " $W$-band micromachined circuit combining networks," IEEE Trans. Microw. Theory Tech., vol. 50, no. 6, pp. 1647-1651, Jun. 2002.

[4] S. Horst, S. Bhattacharya, M. M. Tentzeris, and J. Papapolymerou, "Monolithic low cost $K a$-band Wilkinson power dividers on flexible organic substrates," in Electron. Compon. Technol. Conf., Jun. 2007, pp. $1851-1854$.

[5] S. Song, C. S. Yoo, D. Kim, S. Choi, J. C. Park, and K. S. Seo, "Development of millimeter-wave integrated passives on MCM-D technology with thin-film microstrip line," in IEEE MTT-S Int. Microw. Symp. Dig., Jun. 2006, pp. 952-955.

[6] L. H. Lu, Y. T. Liao, and C. R. Wu, "A miniaturized Wilkinson power divider with CMOS active inductors," IEEE Microw. Wireless Compon. Lett., vol. 15, no. 11, pp. 775-77, Nov. 2005.

[7] D. Antsos, "Modified Wilkinson power dividers for $K$ - and K $a$-bands," Microw. J., vol. 38, no. 11, pp. 98-104, Nov. 1995.

[8] R. Garg and I. J. Bahl, "Characteristics of coupled microstriplines," IEEE Trans. Microw. Theory Tech., vol. MTT-27, no. 7, pp. 700-705, Jul. 1979

[9] D. C. Thompson, O. Tantot, H. Jallageas, G. E. Ponchak, M. M. Tentzeris, and J. Papapolymerou, "Characterization of liquid crystal polymer (LCP) material and transmission lines on LCP substrates from 30 to $110 \mathrm{GHz}$," IEEE Trans. Microw. Theory Tech., vol. 52, no. 4, pp. 1343-1352, Apr. 2004.

[10] S. Horst, S. Bhattacharya, S. Johnston, M. M. Tentzeris, and J. Papapolymerou, "Modeling and characterization of thin film broadband resistors on LCP for RF applications," in 56th Electron. Compon. Technol. Conf., Jun. 2006, pp. 1751-55.

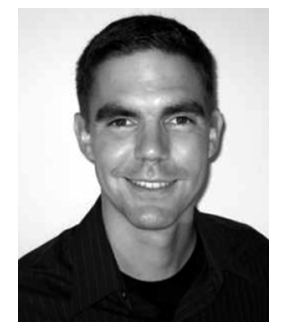

Stephen Horst (S'00) received the B.S. degree (with distinction) in electrical and computer engineering from The Ohio State University, Columbus, in 2004, the M.S. degree in electrical and computer engineering from the Georgia Institute of Technology, Atlanta, in 2006, and is currently working toward the Ph.D. degree at the Georgia Institute of Technology. His B.S. thesis concerned subharmonic superposition in radar IF receivers. His M.S. thesis concerned low-cost fabrication of millimeter-wave circuits using LCP substrates.

From 2001 to 2003, he was an Intern with Cisco Systems, Akron, OH, where he was involved with RF circuit and system design in UNII-1 and UNII-2 unlicensed bands. He is currently involved in the study of frequency synthesis techniques on silicon germanium. 


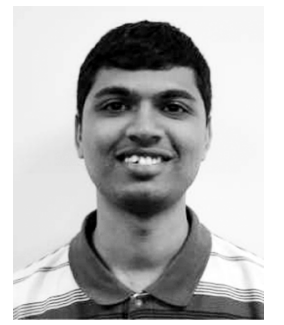

Ramanan Bairavasubramanian ( $\mathrm{S}^{\prime}$ '02) received the B.E. degree in electrical and electronics engineering from Anna University, Chennai, India, in 2001 and the M.S. and Ph.D. degrees in electrical and computer engineering from the Georgia Institute of Technology, Atlanta, in 2002 and 2007, respectively. His doctoral dissertation focused on the development of microwave and millimeter-wave antennas and passive components on multilayer LCP technology.

$\mathrm{He}$ is currently a Design Engineer with Skyworks Solutions Inc., Cedar Rapids, IA. His current research interests include development of system-on-a-chip and system-on-a-package transceiver front-end architectures for mobile communication applications.

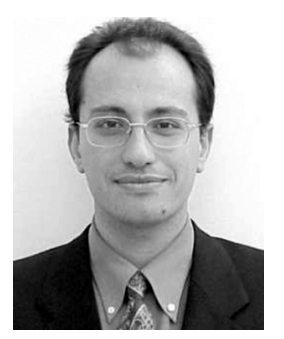

Manos M. Tentzeris (SM'03) received the Diploma degree in electrical and computer engineering from the National Technical University of Athens, Athens, Greece, in 1992, and the M.S. and Ph.D. degrees in electrical engineering and computer science from The University of Michigan at Ann Arbor, in 1993 and 1998, respectively.

$\mathrm{He}$ is currently an Associate Professor with the School of Electrical and Computer Engineering, Georgia Institute of Technology, Atlanta. He has authored or coauthored over 260 papers in refereed journals and conference proceedings, two books, and ten book chapters. He has helped develop academic programs in highly integrated/multilayer packaging for RF and wireless applications, microwave microelectromechanical systems (MEMS), system-on-package (SOP) integrated antennas and adaptive numerical electromagnetics (finite difference time domain (FDTD), multiresolution algorithms), and heads the ATHENA Research Group (15 researchers). He is the Georgia Institute of Technology National Science Foundation (NSF) Packaging Research Center Associate Director for RF Research and the RF Alliance Leader. He is also the leader of the Novel Integration Techniques Subthrust of the Broadband Hardware Access Thrust of the Georgia Electronic Design Center (GEDC) of the State of Georgia. During the summer of 2002, he was a Visiting Professor with the Technical University of Munich, Munich, Germany, where he introduced a course in the area of high-frequency packaging. He has given over 40 invited talks in the same area to various universities and companies in Europe, Asia, and the U.S.

Dr. Tentzeris is a member of URSI Commission D, an associate member of EuMA, and a member of the Technical Chamber of Greece. He was the 1999 Technical Program co-chair of the 54th ARFTG Conference, Atlanta, GA, and he is the vice-chair of the RF Technical Committee (TC16) of the IEEE Components, Packaging, and Manufacturing Technology (CPMT) Society. He has organized various sessions and workshops on RF/Wireless Packaging and Integration in IEEE ECTC, IMS, and AP-S Symposia, for all of which he is a member of the Technical Program Committee in the area of components and RF. He was the recipient of the 2003 National Aeronautics and Space Administration (NASA) Godfrey "Art" Anzic Collaborative Distinguished Publication Award for his activities in the area of finite-ground low-loss low-crosstalk coplanar waveguides (CPWs), the 2003 IBC International Educator of the Year Award, the 2003 IEEE CPMT Outstanding Young Engineer Award for his work on 3-D multilayer inte- grated RF modules, the 2002 International Conference on Microwave and Millimeter-Wave Technology Best Paper Award (Beijing, China) for his work on compact/SOP-integrated RF components for low-cost high-performance wireless front-ends, the 2002 Georgia Institute of Technology Electrical and Computer Engineering Outstanding Junior Faculty Award, the 2001 ACES Conference Best Paper Award, the 2000 NSF CAREER Award for his work on the development of multiresoluation time-domain (MRTD) technique that allows for the system-level simulation of RF integrated modules, and the 1997 Best Paper Award of the International Hybrid Microelectronics and Packaging Society for the development of design rules for low-crosstalk finite-ground embedded transmission lines. He was the recipient/corecipient of the 2007 IEEE AP-S Symposium Best Student Paper Award, the 2007 IEEE IMS Third Best Student Paper Award, the 2007 ISAP 2007 Second Best Poster Presentation Award, the 2006 IEEE MTT-S Outstanding Young Engineer Award, and the 2006 Asia-Pacific Microwave Conference Award.

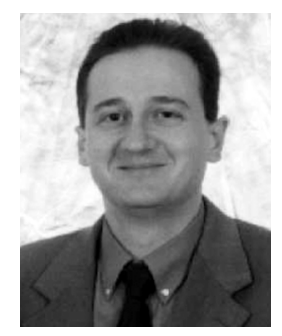

John Papapolymerou (S'90-M'99-SM'04) received the B.S.E.E. degree from the National Technical University of Athens, Athens, Greece, in 1993, and the M.S.E.E. and Ph.D. degrees from The University of Michigan at Ann Arbor, in 1994 and 1999, respectively.

From 1999 to 2001, he was an Assistant Professor with the Department of Electrical and Computer Engineering, University of Arizona, Tucson. During the summers of 2000 and 2003, he was a Visiting Professor with the University of Limoges, Limoges, France. From 2001 to 2005, he was an Assistant Professor with the School of Electrical and Computer Engineering, Georgia Institute of Technology, Atlanta, where he is currently an Associate Professor. He has authored or coauthored over 140 publications in peer-reviewed journals and conferences. His research interests include the implementation of micromachining techniques and MEMS devices in microwave, millimeter-wave and terahertz circuits and the development of both passive and active planar circuits on semiconductor ( $\mathrm{Si} / \mathrm{SiGe}$, $\mathrm{GaAs}$ ) and organic substrates [liquid-crystal polymer (LCP), low-temperature co-fired ceramic (LTCC)] for system-on-a-chip (SOC)/system-on-package (SOP) RF front ends.

Dr. Papapolymerou is the vice-chair for Commission D of the U.S. National Committee of URSI. He is an associate editor for IEEE MICROWAVE AND WIRELESS COMPONENT LETTERS and the IEEE TRANSACTIONS ON ANTENNAS AND PROPAGATION. During 2004, he was the chair of the IEEE Microwave Theory and Techniques (MTT)/Antennas and Propagation (AP) Atlanta Chapter. He was the recipient of the 2004 Army Research Office (ARO) Young Investigator Award, the 2002 National Science Foundation (NSF) CAREER Award, the Best Paper Award presented at the 3rd IEEE International Conference on Microwave and Millimeter-Wave Technology (ICMMT2002), Beijing, China, and the 1997 Outstanding Graduate Student Instructional Assistant Award presented by the American Society for Engineering Education (ASEE), The University of Michigan at Ann Arbor Chapter. His student was also the recipient of the Best Student Paper Award presented at the 2004 IEEE Topical Meeting on Silicon Monolithic Integrated Circuits in RF Systems, Atlanta, GA. 